\title{
Révélation de ressources spécifiques et coordination située
}

Gabriel Colletis et Bernard Pecqueur

\section{(2) OpenEdition \\ 12 Journals}

Édition électronique

URL : http://journals.openedition.org/ei/900

DOI : 10.4000/ei.900

ISSN : 2553-1891

Éditeur

Association Économie et Institutions

Édition imprimée

Date de publication : 1 décembre 2005

Pagination : $51-74$

ISSN : 1775-2329

Référence électronique

Gabriel Colletis et Bernard Pecqueur, "Révélation de ressources spécifiques et coordination située »,

Économie et institutions [En ligne], 6-7 | 2005, mis en ligne le 31 janvier 2013, consulté le 01 mai 2019. URL : http://journals.openedition.org/ei/900 ; DOI : 10.4000/ei.900 


\title{
Révélation de ressources spécifiques et coordination située
}

\author{
Gabriel COLLETIS ${ }^{1}$ et Bernard PECQUEUR ${ }^{2}$
}

Les évolutions socio-économiques du dernier quart de siècle, notamment pour ce qui est des transformations complexes liées au processus dit de "mondialisation", reposent avec acuité les problèmes de division "internationale" du travail et des inégalités de création et répartition de richesses à l'échelle mondiale. L'interdépendance des marchés, la mondialisation financière et le mouvement de délocalisation/ relocalisation des grandes entreprises constituent des phénomènes concrets qui illustrent ces évolutions. Dans l'actualité récente, les délocalisations brutales (une usine déménagée dans la nuit, l'apparition de la notion de "patron voyou",...) viennent confirmer que les mouvements à l'œuvre exacerbent l'apparente tendance à l'a-spatialité du capitalisme contemporain. L'organisation de la production se déploierait sur des espaces interchangeables qui ne seraient que des supports plus ou moins coûteux en facteurs de production. Cette vision non géographique d'une mondialisation envahissante doit, bien sûr, être corrigée par l'ensemble des débats qui soulignent que les hiérarchies spatiales demeurent, de même que, par conséquent, demeurent des centres et des périphéries, des cycles du produit où la technologie serait inégalement "répartie" entre les lieux où elle se produit et ceux où elle se diffuse. Cependant, les approches qui sous-tendent ces débats adoptent une représentation qui peut être contestée du changement technologique et nient la notion de proximité des agents ${ }^{3}$ ainsi que l'importance que revêt aujourd'hui la construction d'espaces économiques par les acteurs qui créent ces espaces (territoires). L'idée selon laquelle les espaces géographiques sont des lieux de création de ressources, lesquelles doivent être distinguées de celles qu'utilisent et que génèrent les entreprises implantées dans ces espaces, est relativement nouvelle et nous semble se diffuser progressivement ${ }^{4}$.

\footnotetext{
1 LEREPS, Université Toulouse 1, Gabriel.Colletis@univ-tlse1.fr.

2 PACTE, Université Grenoble 1, Bernard.Pecqueur@ujf-grenoble.fr

3 Cf. l'ouvrage collectif : B. Pecqueur et J.-B. Zimmermann (dir.) : Economie de proximités, éditions Hermès-Lavoisier, 2004.

4 On lira notamment : G. Benko et A. Lipietz (2000) mais aussi les travaux sur les "learning regions" (D. Maillat, O. Crevoisier, L. Kébir,...) qui approfondissent la notion de milieu innovateur développée par le GREMI (Groupe de Recherche Européen sur les Milieux Innovateurs). Voir notamment Kébir (2004).
} 
Nous avons proposé (Colletis et Pecqueur, 1993 et 1995) une réflexion sur la nature des ressources produites et valorisées en intégrant l'origine et la nature de ces ressources. Pour cela nous avons utilisé et fortement réinterprété la notion williamsonnienne d'actif spécifique. Cette notion, issue donc de la Nouvelle Economie Institutionnelle, dépasse en fait la seule organisation de coordinations visant à pallier les imperfections du marché, pour constituer un des fondements d'un véritable modèle de développement territorial.

Plus de dix ans après avoir proposé cette notion de spécificité et la distinction entre une ressource qui existe à l'état latent ou virtuel et sa valorisation ou révélation sous forme d'actif, nous cherchons ici à actualiser nos définitions et à montrer en quoi la notion de ressource/actif spécifique est plus que jamais un outil méthodologique fécond pour appréhender aujourd'hui les enjeux de la mondialisation et de la territorialisation des processus productifs. Cette territorialisation est le corollaire, selon nous, d'une mondialisation davantage fondée sur l'approfondissement de la diversité (diversité des trajectoires socio-économiques des différents espaces et territoires, diversité des "modèles" productifs, etc.) que sur une supposée tendance générale à l'homogénéisation (celle-ci pouvant s'exprimer cependant dans la production de telle ou telle norme).

Dans une première partie de cet article, nous repartons de notre point de départ, à savoir de notre proposition de grille d'analyse de ce que nous avons désigné comme les "facteurs de concurrence spatiale" (Colletis et Pecqueur, 1993), en apportant un certain nombre de précisions sur les notions de ressource et de spécificité. Notre approche est également resituée dans un ensemble de travaux qui ont, selon nous, comme dénominateur commun de raisonner (parfois implicitement) en termes de création de ressources et de production d'externalités.

Dans une seconde partie, nous proposons de tirer un certain nombre de conséquences d'une approche résolument dynamique de processus de coordination situés dans le temps mais aussi dans l'espace. En particulier, nous tentons de montrer que ces processus peuvent être synonymes de construction ou de révélation du territoire et qu'ils peuvent être utilement éclairés par les notions de "patrimoine" (Pecqueur, 2002 ; Pecqueur et Peyrache-Gadeau, 2002) mais aussi de ressource spécifique. 


\section{Une analyse centrée sur la production de ressources}

Nous commencerons par donner un bref aperçu de travaux se situant dans une perspective de création de ressources et de production d'externalités.

Depuis les travaux de Philippe Aydalot (1985) en France, la région au sens large et le territoire, au sens plus étroit ou précis d'un espace construit, sont revenus au cœur des préoccupations d'analyse de "l'agglomération spatiale". La modernité d'A. Marshall apparaît avec la lecture italienne des districts italiens (Becattini, 1977 ; Courlet, 2000). Elle situe la problématique des ressources en termes de création de ressources et de production d'externalités par les acteurs.

La nouveauté de ces approches repose sur le lien organique qui est suggéré entre processus d'innovation et de développement, d'une part, ancrage territorial de ces processus, d'autre part. La notion "d'agglomération de l'innovation" illustre assez bien ce lien.

Ainsi, pour Gay et Picard $(2001)^{5}$, le "territoire vécu, organisé, où peuvent s'exprimer pouvoir, stratégie et confiance, offre une configuration idéale pour l'émergence des activités d'innovation en servant de catalyseur aux interactions qui les engendrent (...) Last but not least, la capacité innovante attachée au territoire en fait un instrument privilégié de développement endogène qui justifie sa mobilisation dans l'action publique". Aujourd'hui, c'est donc en partie dans la perspective d'une problématique en termes de "géographie de l'innovation" qu'est traitée la question de "l'agglomération" de l'innovation.

En matière d'analyse des phénomènes d'innovation technologique, la littérature a fortement convergé vers une lecture plus "territorialisée" du phénomène. A partir d'une approche située dans un cadre national, avec les "Système Nationaux d'Innovation", on est ainsi passé aux "Systèmes Régionaux d'Innovation" (Cooke et Morgan, 1998), en approfondissant l'analyse des différenciations spatiales qui transcenderaient l'organisation nationale des systèmes productifs. Scott (2001) pose pour sa part, en forme de provocation, la question de savoir si le capitalisme va "vers la désagrégation des économies nationales". Le problème de "l'entité étatique", du moins comme unité spatiale homogène du développement, semble posé, même si ce niveau "d'agrégation" ou de cohérence socio-économique, à l'évidence, "résiste".

Cependant, les transformations liées à la mondialisation nous semblent exiger de devoir pousser le raisonnement de la possible

5 On trouvera une synthèse de ces débats autour notamment de la notion d'externalités technologiques de connaissance (knowledge spillovers) et leur ancrage spatial dans C. Gay et F. Picard (2001). 
déconstruction de l'image d'un Etat-nation homogène plus loin que la seule proposition de "solution" en termes de "regroupement(s) régional(aux)".

Dans un contexte particulier, en partie lié à son histoire propre, le monde (acteurs privés et publics "réels" comme chercheurs) anglo-saxon s'est emparé de la notion de clusters pour désigner des "sites"6, lieux d'unité ou d'unification de stratégie d'acteurs et de développement de ressources particulières. On retiendra, par ailleurs, de ce bref aperçu de ce vaste débat, l'idée que la ville constitue un espace à part entière, un milieu urbain générateur d'externalités propres ${ }^{7}$. La ville constitue un bon exemple de "site" comme espace-lieu significatif d'une élaboration de type territorial. Camagni (1999) le rappelle : "le milieu et la ville partagent des éléments communs, notamment la proximité, porteurs non seulement d'avantages en termes d'économies d'échelle et de réduction des coûts de transaction, mais aussi, sous certaines conditions, d'avantages à caractère dynamique qui se manifestent dans l'apprentissage, l'innovation économique et sociale, la créativité en général" (Camagni, 1999). Ainsi les territoires ne sauraient être analysés comme de simples regroupements spatiaux infranationaux de taille variable mais plutôt comme des entités socialement construites résultant de processus particuliers de coordination d'acteurs. Ces entités fonctionnent comme composantes actives des reconfigurations actuelles de l'économie mondiale.

\subsection{Concurrence des territoires et spécificité}

L'analyse économique, et l'économie industrielle en particulier, accordent une place centrale à la compréhension des formes et mécanismes de la concurrence. La concurrence par les coûts et les prix constitue le noyau dur de l'analyse ainsi que l'hypothèse de référence des schémas de concurrence (concurrence pure et parfaite, concurrence oligopolistique). Des travaux empiriques de qualité ont cependant montré, au niveau des économies nationales, que la maîtrise des coûts ne suffisait pas à assurer un commerce extérieur équilibré ou excédentaire (Mathis, Mazier et Rivaud-Danset, 1988).

Au niveau des entreprises, la maîtrise des coûts confère un avantage concurrentiel important, mais qui, seul, peut se révéler

6 Voir C. Gay et F. Picard (2001) mais aussi les travaux de D.B. Audretsch et M. P. Feldman (1996) sur les clusters de bio-technologie; voir également les travaux de N. Massard, notamment in N. Massard, A. Torre et O. Crevoisier (2004).

7 On se réfère aux travaux fondateurs de J. Rémy (1966). Voir également S. Sassen (1991) ou encore, plus récemment, R. Guillain et J.-M. Huriot (2000) ainsi que L. Bourdeau-Lepage et J.-M. Huriot (2003).

54 Economie et Institutions $-n^{\circ} 6$ et $7-1^{\text {er }}$ et $2^{\text {e }}$ semestres 2005 
insuffisant. La capacité d'innover, de répondre rapidement aux variations de la demande en volume, de proposer des produits correspondants aux besoins spécifiques des demandeurs constitue aujourd'hui autant de variables essentielles des stratégies concurrentielles, parfois regroupées sous le terme quelque peu imprécis de "flexibilité" (Piore et Sabel, 1984).

L'accent mis sur les formes nouvelles de la concurrence, et notamment sur la concurrence par la différenciation de l'offre, peut conduire à proposer une typologie des facteurs de concurrence rendant compte de cette différenciation et qui ne soit pas nécessairement a priori marquée par un choix préalable exclusif du champ de cette concurrence: concurrence entre espaces économiques (pays ou régions) ou concurrence entre firmes. Ce type de réflexion se situe ainsi en amont méthodologique des analyses relatives aux facteurs de localisation des entreprises, qui distinguent plus qu'elles n'articulent les variables relatives aux stratégies des firmes et celles liées aux pays ou régions d'implantation.

\subsection{Les bases d'une double distinction actif/ressource et facteurs à caractère générique/spécifique}

La typologie que nous utilisons $\mathrm{ici}^{8}$ consiste à distinguer, d'une part, actif et ressource, d'autre part, à qualifier les actifs ou les ressources selon leur nature, générique ou spécifique.

Par actif, on entendra des facteurs "en activité", alors que par ressources il s'agira de facteurs à exploiter, à organiser, ou encore à révéler. Les ressources, à la différence des actifs, constituent une réserve, un potentiel latent ou virtuel qui peut se transformer en actif si les conditions de production ou de création de technologie le permettent.

Des actifs ou des ressources génériques se définissent par le fait que leur valeur ou leur potentiel sont indépendants de leur participation à un quelconque processus de production. Les actifs ou ressources sont ainsi totalement transférables, leur valeur est une valeur d'échange. Le lieu de cet échange est le marché. Le prix est le critère d'appréciation de la valeur d'échange, laquelle est déterminée par une offre et une demande à caractère quantitatif. En d'autres termes, un facteur générique est indépendant du "génie du lieu" où il est produit.

Par contre, les actifs spécifiques, existent comme tels, mais leur valeur est fonction des conditions de leur usage. Alors qu'un actif générique est totalement transférable, un actif spécifique implique un coût irrécouvrable plus ou moins élevé de transfert.

Les ressources spécifiques n'existent qu'à l'état virtuel et ne

${ }^{8}$ pour une première formulation, voir G. Colletis et B. Pecqueur, 1993.

Economie et Institutions $-n^{\circ} 6$ et $7-1^{\text {er }}$ et $2^{\text {e }}$ semestres 2005 
peuvent en aucun cas être transférées. Ces ressources naissent de processus interactifs et sont alors engendrées dans leur configuration. Elles constituent l'expression du processus cognitif qui est engagé lorsque des acteurs ayant des compétences différentes produisent des connaissances nouvelles par la mise en commun de ces dernières. Lorsque des connaissances et savoirs hétérogènes sont combinés, de nouvelles connaissances sont produites qui peuvent, à leur tour, participer de nouvelles configurations. La création de technologie est ainsi le résultat d'un processus caractérisé par l'émergence de ressources spécifiques issues d'une dynamique cognitive synonyme d'un apprentissage interactif.

Des actifs génériques ne permettent pas à un territoire de se différencier durablement puisque, par définition, ils existent ailleurs et sont susceptibles d'être transférés. Une différenciation durable, c'est-à-dire non susceptible d'être remise en cause par la mobilité des facteurs, ne peut naitre véritablement que des seules ressources spécifiques, lesquelles ne sauraient exister indépendamment des conditions dans lesquelles elles sont engendrées.

L'enjeu des stratégies de développement des territoires est donc essentiellement de saisir ces conditions et de rechercher ce qui constituerait le potentiel identifiable d'un territoire (désigné plus loin par la notion de "patrimoine"). Ces conditions ne sauraient être définies de façon abstraite. Elles dépendent du contexte dans lequel s'inscrit le processus heuristique d'où naissent les ressources spécifiques.

\subsection{Ressources et actifs génériques}

Le qualificatif "générique" recouvre l'ensemble des facteurs traditionnels de définition spatiale (ou de localisation des activités économiques) discriminés par les prix (dont les coûts de transport) et qui font l'objet de la part des agents d'un calcul d'optimisation.

On illustrera les situations de ressources ou d'actifs génériques à l'aide de quatre exemples : la main-d'œuvre, le capital, la matière première et l'information. Ces quatre facteurs resteront à l'état de ressources génériques dans les cas suivants: la maind'œuvre est non qualifiée et non utilisée. Il existe un stock de capital sous forme d'épargne mais celle-ci est totalement liquide ou non utilisée (thésaurisée). Les gisements de matière première existent mais ne sont pas exploités. L'information est disponible sous forme standard mais n'est pas utilisée (interprétée) dans un contexte particulier. C'est le cas d'informations contenues dans une banque de données, d'une encyclopédie ou d'un catalogue...encore que ces "informations" sont, en réalité, des connaissances en ce qu'elles véhiculent des représentations.

Ces ressources, en devenant des actifs, ne cessent pas pour

56 Economie et Institutions $-n^{\circ} 6$ et $7-1^{\text {er }}$ et $2^{\text {e }}$ semestres 2005 
autant d'être génériques. Elles ne changent pas de nature mais elles sont "réalisées" et acquièrent ainsi une valeur économique, valeur pouvant dans certaines conditions être mesurée en prix. La maind'œuvre reste non qualifiée mais est employée. Le chômeur non qualifié représente un actif et pas simplement une ressource car, même si son travail n'est pas réellement utilisé, il est présent et influe sur le marché du travail et éventuellement donc sur le taux de salaire. Le capital prêt à s'investir se révèle et devient épargne active. Cette épargne reste très liquide et ne peut donc être affectée qu'à des placements à court terme. La matière première est mise en exploitation. L'information, sous sa forme standard, est effectivement utilisée avant de participer, éventuellement, comme ressource, à un processus de construction de connaissance. Du point de vue métaphorique, l'encyclopédie est une ressource (elle se consulte), le livre est un actif (il se lit).

Les actifs génériques sont totalement dans le marché. C'est-àdire que, pour les acquérir, il existe un prix de marché. Ils sont totalement transformables, immédiatement disponibles, à condition de s'acquitter de leur prix. Pour ces actifs, il n'existe pas de contrainte géographique (hors de la matière première mais cette dernière est substituable). Ajoutons enfin que leur acquisition ne nécessite pas l'existence et l'activation de formes de coordination partiellement hors marché comme, par exemple, les réseaux.

\subsection{Actifs et ressources spécifiques}

La relation entre actifs et ressources, dans ce cas, est plus complexe que dans le cas précédent. Nous montrerons qu'il y a ici une différence de nature entre l'actif et la ressource. Le qualificatif de spécifique recouvre l'ensemble des facteurs, comparables ou non, dont la valeur ou la production est liée à un usage particulier.

Les actifs spécifiques ont un coût d'irréversibilité que l'on pourrait appeler aussi un "coût de réaffectation". Cela signifie que l'actif perd une partie de sa valeur productive dans le cas où il est redéployé vers un usage alternatif. En reprenant les illustrations utilisées plus haut, on dira que la matière première ne peut pas avoir de caractère spécifique dans la mesure où sa valeur marchande n'est pas liée à son usage futur ni au contexte socioéconomique immédiat. Par contre la main-d'œuvre sera un actif spécifique dès lors qu'elle est qualifiée. Sa formation à une technique constitue un coût pris en charge partiellement hors de l'entreprise. Une délocalisation aurait, en partie, un coût de réaffectation pour l'entreprise utilisatrice de cette main-d'œuvre. De même, le capital devient actif spécifique lorsqu'il passe d'une forme d'épargne liquide à une forme de capital investi dans des équipements. Enfin, l'information devient, elle aussi, un actif spécifique lorsqu'elle est élaborée et organisée en vue d'un 
usage particulier. Concrètement, on peut citer l'élaboration de logiciels très techniques concentrant l'information pour servir une production très pointue liée à des usages particuliers.

Les ressources spécifiques, sur lesquelles nous revenons en seconde partie de ce papier, ont une nature particulière par rapport aux trois autres catégories de ressources ou actifs. En premier lieu, ces ressources n'apparaissent qu'au moment des combinaisons des stratégies d'acteurs pour résoudre un problème inédit. Plus précisément, la formulation même du problème en même temps que sa solution font l'objet d'un processus heuristique marqué par des tâtonnements et des itérations successives.

En deuxième lieu, ces ressources ne sont pas commensurables, ce qui signifie qu'on ne peut les exprimer en prix et qu'elles sont donc absolument non transférables.

En troisième lieu, la nature partiellement hors marché de ces ressources n'est pas incompatible mais complémentaire au marché. Ces ressources résultent d'une histoire longue, d'une accumulation de mémoire, d'un apprentissage collectif cognitif.

En dernier lieu, ces ressources sont, dans certains cas, produites dans un territoire, lequel est alors "révélé". La production de telles ressources résulte en effet de règles, de coutumes, d'une culture élaborées dans un espace de proximité géographique et institutionnelle à partir d'une forme d'échange distincte de l'échange marchand : la réciprocité. Les caractéristiques de cette réciprocité eu égard au marché sont que si l'échange reste "contraint" (obligation sociale), il peut être différé dans le temps (le délai du retour attendu peut être très variable), enfin, voire surtout, ce retour n'est pas nécessairement monétaire, il peut être confiance, considération, reconnaissance ou savoir. La réciprocité comme terreau des ressources spécifiques est ainsi, le plus souvent, à la base des relations informelles qui composent une "atmosphère industrielle" au sens où l'entendait A. Marshall décrivant certaines concentrations des petites unités industrielles dans l'Angleterre ou l'Allemagne du début du $\mathrm{XIX}^{\circ}$ siècle. Plus systématiquement, le "sentiment d'appartenance", à un lieu ou à un métier, la "culture d'entreprise" ou encore "l'esprit maison", font partie des ressources "spécifiques", dans un esprit proche de celui de Veblen (Veblen, 1899). La ressource spécifique produite lors d'un processus de construction territoriale, prise globalement, apparait alors comme le résultat de processus long d'apprentissages collectifs qui aboutissent à l'établissement de règles, le plus fréquemment tacites. De telles règles ont la fonction de trier, de sélectionner et de hiérarchiser l'information, mais aussi d'orienter ou guider les comportements offrant, ainsi à chaque acteur du territoire un espace d'intelligibilité et d'action.

58 Economie et Institutions $-n^{\circ} 6$ et $7-1^{\text {er }}$ et $2^{\text {e }}$ semestres 2005 


\subsection{L'activation et la spécification : un processus de métamorphose}

Le passage de la ressource générique à l'actif spécifique correspond à une stratégie de développement qui peut se décomposer en deux temps : le passage de la ressource à l'actif (génériques), et de l'actif générique à l'actif spécifique. Ces passages doivent être chacun analysés comme des processus particuliers qui font muter en profondeur la nature même des objets concernés. C'est dans ce sens que l'on parle de métamorphose comme un changement structurel avec une irréversibilité incomplète selon laquelle on ne peut pas toujours revenir de l'actif vers la ressource et du spécifique au générique en retrouvant l'état initial exact.

Afin d'abord de préciser la notion de ressource, nous pouvons faire appel à A.-O. Hirschman (1986). Selon celui-ci, "il importe moins, pour promouvoir le développement économique, de trouver des combinaisons optimales de ressources et de facteurs de production donnés que de faire apparaître et de mobiliser à son service des ressources et des capacités cachées, éparpillées ou mal utilisées". En ce sens la ressource se distingue clairement du facteur de production. Cette dernière notion, basique en économie standard pour désigner les ressources qui entrent comme input dans le processus de production, est en effet très restrictive et limitée au cas de l'actif générique dans notre formulation.

Deux cas "d'activation" peuvent être envisagés : celui d'une ressource (générique) potentielle et pré-existante, celui d'une ressource (spécifique) virtuelle. Dans le premier cas, le marché est le lieu ou le moyen par lequel se produit l'activation de la ressource. Dans le second cas, la ressource virtuelle peut être activée à la suite d'un processus particulier d'engagement. Ainsi, dans une perspective proche de la nôtre, pour Lévy et Lussault (2003) : "une réalité issue $\mathrm{du}$ monde physique ou biologique ne peut être ressource que s'il existe un processus de production identifié dans lequel il peut être inséré et qui, par définition, provient de la société (...) Les ressources sont donc toujours inventées (souligné par nous), parfois bien après avoir été découverte comme le pétrole en tant que source d'énergie ou la haute montagne comme "gisement" touristique".

L. Kébir (2004) définit en des termes proches la notion de ressource comme un "méta-système mettant en relation un objet (savoir faire, matière première, artefact, etc.) et un système de production produisant un bien ou un service".

Il s'agit d'un "méta-système" dans la mesure où il résulte d'une combinaison de deux systèmes préalablement existant : celui de l'objet et celui du système de production ou plutôt, selon nous, de règles qui le réalise (voir plus loin en seconde partie la notion de patrimoine). 
L'objet/ressource qui va servir de support à la production d'un actif ne peut être réduit à sa dimension d'input mais relève d'un système qui lui est propre : "avant de fournir une planche, un arbre est un arbre. Ceci même lorsqu'il est planté à cette fin" (Kébir, 2004). La même perspective peut également être retenue s'agissant des ressources immatérielles que constituent les paysages, les traditions patrimoniales, les savoir faire, voire le capital social. Ainsi l'objet va subir sa métamorphose à travers le système de règles qui le transforme en ressource activée. "Le système de production (de règles, selon nous) est le lieu de l'identification (souligné par l'auteur) et de la mise en ouvre des ressources. (...) Elles y sont actualisées, "transformées et utilisées " (Kébir, 2004). En d'autres termes, la ressource en devenant actif est transformée par le système de production (pour nous, de règles) et change de nature et au stade de la virtualité, tout peut potentiellement faire ressource mais tout ne peut devenir mécaniquement un actif puisque les conditions de la métamorphose ne sont pas dans l'objet/ressource mais dans les stratégies d'acteurs au sein du processus de production.

Ainsi, les ressources se déploient et se transforment, mais aussi régressent ou redeviennent un potentiel, selon qu'elles évoluent en actifs ou, d'actifs, reviennent au statut de ressources. Cependant, comme nous l'avons suggéré, le processus de métamorphose qui fait passer la ressource au statut d'actif n'a pas le même sens selon qu'il s'agit de ressources génériques ou spécifiques. Dans le cas de la ressource générique, reproductible en tout lieu, ce qui peut faire régresser l'actif de nouveau vers la ressource est uniquement fonction des coûts. On vient de fermer le dernier puits de charbon en Lorraine mais on n'a pas pour autant ôté le charbon. Celui-ci retourne au statut de ressource dans l'état où il était avant l'ouverture de la mine et, dans le cas d'une hypothétique réduction des coûts d'exploitation, cette ressource pourrait redevenir un actif (exploité).

La spécificité a, comme nous l'avons vu en partie, des caractéristiques particulières qui méritent d'être plus nettement précisées. C'est ce que nous nous proposons de faire à présent en rapport avec une approche dynamique de la coordination.

\section{Pour une approche de la coordination "en dynamique"}

A l'évidence, la question de la coordination et, plus encore, la façon dont celle-ci est abordée, sont centrales pour appréhender le territoire en "dynamique".

La première question est ainsi au centre de l'intitulé des Journées "Proximité, réseaux et coordination" (Marseille, 17-18 juin 2004) comme elle est au centre de certaines communications qui

60 Economie et Institutions $-\mathrm{n}^{\circ} 6$ et $7-1^{\mathrm{er}}$ et $2^{\mathrm{e}}$ semestres 2005 
seront présentées pendant ces Journées (notamment Billaudot, 2004) ou encore de textes plus ou moins récents. ${ }^{9}$

C'est donc cette question de la coordination que nous souhaitons aborder à présent en la situant "en dynamique", c'est-àdire en explicitant ce que signifie selon nous de poser la question de la coordination en l'inscrivant dans une perspective où l'accent est mis sur les processus de construction du territoire et non sur l'hypothèse d'un territoire "existant" ou postulé pour ou par l'analyse.

On en déduira des caractéristiques particulières au territoire résultant de la typologie présentée dans la première partie de ce texte. Ces caractéristiques sont au nombre de trois : le territoire apparaît comme un espace "révélé", les ressources du territoire sont celles qui constituent son "patrimoine", la proximité géographique demeure une catégorie fondatrice du territoire même si celui-ci ne peut se limiter à elle.

\subsection{Un territoire révélé}

Dès lors que l'on se situe dans cette perspective, aux notions de territoire "doté" et même de territoire "institué" ou "approprié", nous préférons ou, plus radicalement, nous substituons celle de territoire "révélé" (Colletis et Pecqueur, 1993).

La notion de territoire révélé suggère que, comme tel, le territoire économique ou industriel n'existe pas et qu'il est révélé en dynamique dans et par des situations de coordination situées dans le temps et aussi dans l'espace.

Cela souligne l'importance de dynamiques que nous avons qualifiées précédemment "d'activation" et de "spécification" (Colletis et Pecqueur, 1993; Colletis, Pecqueur et Gilly, 2001).

D'où également notre approche particulière de la notion de ressource dont l'attribut principal en dynamique est le caractère potentiel ou latent (ressources génériques, susceptibles d'être activées) ou encore virtuel (ressources spécifiques, ressources collectives non rivales n'apparaissant qu'au moment de la combinaison originale de compétences complémentaires en vue de résoudre un problème inédit) (Colletis et Pecqueur, 1993 et 1995).

Notre approche, comme on l'aura compris, s'inspire de ce point de vue de celle de. Hirschman (1986) pour ce qui est de l'importance de la "latence", ou encore de celle de Lancaster (1991) pour ce qui est de la distinction entre biens (qui comme tels n'apportent aucune satisfaction) et activités (dans lesquelles les

9 Cf. notamment l'introduction de l'ouvrage coordonné par B. Pecqueur et J.B. Zimmermann (2004), ou encore G. Colletis (1998). 
biens sont des ingrédients) pour souligner l'importance $d u$ "contexte", de la" mise en situation".

Une lecture en termes de territoire "doté" ou encore de territoire comme "réservoir", "stock" ou même "conteneur" de ressources est souvent proposée. Dans ce cas, le territoire est "donné" ou "borné". Une telle représentation peut s'appuyer sur l'existence d'espaces politico-administratifs de type région, départements ou cantons.

Même si l'on est souvent amené à raisonner sur ce type de territoire, nous analysons ici le territoire construit, c'est-à-dire celui qui n'existe pas ex ante mais qui s'élabore ex post.

Nous situons donc notre approche comme réfutant tout aussi bien la vision d'un territoire doté comme celle d'un territoire-néant, l'enjeu étant alors de dire quelle est la nature des ressources sur la base desquelles le processus de construction du territoire peut s'opérer.

De ce point de vue, la distinction ressources d'allocation (mobilisées dans les activités proprement dites) et ressources d'autorité (mobilisées dans les relations permettant d'intégrer ces activités) proposée par Giddens (1987) est ici éclairante à condition de ne pas considérer les premières comme existantes en l'état.

Les ressources d'autorité, à la différence des ressources d'allocation, ces dernières étant matérielles ou physiques mais aussi des artefacts créés par l'interaction, dérivent, selon Giddens, de l'emprise que certains acteurs ont sur d'autres acteurs. Elles décrivent ainsi la capacité de contrôler ou influencer les activités des êtres humains. L'organisation et le sens de l'espace-temps social, ici le territoire, dépendent ainsi d'abord des ressources d'autorité alors que les caractéristiques de l'environnement dépendent, elles, des ressources d'allocation.

\subsection{Patrimoine et ressources cognitives spécifiques}

Avant d'aller plus loin dans l'analyse des différents types de ressources, nous souhaitons établir le lien entre ressources et "patrimoine", à la suite des travaux de Billaudot (2004) et ceux de C. Barrère et alii $^{10}$. A la différence d'un capital qui relève de la destruction et de l'appropriation, un patrimoine, selon B. Billaudot, relève de la "conservation" et de la "transmission". Un patrimoine serait composé de ressources libres (ressources-externalités et ressources-produits) créées, disponibles ou reproduites dans une structure sociale en raison des activités qui s'y déroulent.

10 C. Barrère, D. Barthélémy, H. Ollagnon, M. Nieddu, F.-D. Vivien (2001) ;

C. Barrère, D. Barthélémy, M. Nieddu, F.-D. Vivien (dir.) (2005).

62 Economie et Institutions $-n^{\circ} 6$ et $7-1^{\text {er }}$ et $2^{\text {e }}$ semestres 2005 
Une ressource-externalité serait ainsi une externalité positive (un effet non visé positif d'une activité sur une autre) constituée en ressource; alors qu'une ressource-produit libre serait un produit (converti en ressource) de libre accès (pour lequel il n'est pas nécessaire d'établir une relation de circulation avec le producteur pour en disposer).

$\mathrm{Au}$ même titre que l'organisation, le territoire serait ainsi (une structure sociale) "titulaire" d'un patrimoine, et la proximité se définirait comme l'attribut d'une personne concernant son accès à un patrimoine (celui en l'occurrence du territoire, ou de l'organisation).

Patrimoine et ressources (et singulièrement ressourcesexternalités) ainsi définis et articulés permettraient ainsi de qualifier un territoire et par delà la proximité.

Le caractère dynamique de cette approche s'exprimerait par la compréhension d'une ressource-externalité qui n'aurait ainsi d'existence que "contextualisée", et ne devrait donc pas son statut à de quelconques ou supposées propriétés intrinsèques. Dans d'autres textes (Colletis et Pecqueur, 1993 et 1995, notamment), nous avions qualifié ces ressources à haute valeur contextuelle de "spécifiques".

Plutôt que de "transmission" (de connaissances tacites) par observation et imitation, et, a fortiori de "conservation", on peut sans doute parler de création de connaissances nouvelles (pour résoudre des problèmes inédits).

En d'autres termes, nous considérons que le "patrimoine"- si l'on veut utiliser ce terme pour aider à qualifier une construction territoriale qui ne produirait pas ex-nihilo - est constitué par la mémoire de situations de coordination antérieures réussies, par la confiance entre les acteurs qui en est le résultat, ainsi que par des ressources cognitives spécifiques virtuellement complémentaires (susceptibles d'être combinées pour résoudre des problèmes productifs à venir).

Les ressources cognitives spécifiques virtuellement complémentaires pourraient être assimilables à des ressources d'allocation. La mémoire de situations de coordination antérieures réussies et la confiance qui en résulte constitueraient le fondement des ressources d'autorité.

Distingué du capital en ce que ce dernier suggère une notion de stock ou d'accumulation, le patrimoine est une notion qui peut se prêter à une utilisation en dynamique en dépit de son acception triviale qui peut être associée à un bien existant comme tel et comme tel transmissible.

Une conception résolument dynamique du territoire est celle d'une institution qui existe par sa capacité non à détenir mais à produire ou créer des ressources. Les seules ressources dont le territoire dispose (et non détient ou contient) sont des ressources liées à une trace d'activités de coordination passées (mémoire, 
confiance) et à un potentiel, une latence, ou encore une virtualité de nature cognitive qui demandent à être activées ou révélée à la faveur d'un problème productif (cf. supra). Nous retrouvons ici la combinaison nécessaire entre ressources d'allocation (les ressources potentielles ou latentes) et ressources d'autorité (la mémoire, la confiance et, de manière générale, les liens entre les hommes, éventuellement investis par des relations de pouvoir).

Si c'est bien la "densité institutionnelle" d'un espace qui en fait un territoire (Abdelmalki et al., 1996), si les processus de spécialisation et de spécification sont toujours basés sur des effets d'accumulation (Olivier et Wallet, 2004) ${ }^{11}$, nous croyons utile de tenter d'éclairer ces processus et ces effets en appréhendant ce que peuvent nous apporter les notions de capital social et de patrimoine.

La notion de capital social semble s'être diffusée à partir des travaux de Coleman (1988). Selon Coleman, le capital social se définit par sa "fonction" qui est de faciliter l'action et les interactions entre individus. Ce capital existe sous trois formes : les obligations et les attentes des individus dans une unité sociale ; l'acquisition, la circulation et le coût de l'information qui est véhiculée par des relations sociales et qui lui confèrent sa crédibilité ; les normes qui engagent et dont la transgression entraîne des sanctions, normes qui assurent ainsi un contrôle social évitant le recours à des procédures formelles.

Le capital social est ainsi davantage défini par ce qu'il fait ou permet que par ce qu'il est.

Différents auteurs ont pu ainsi montrer les limites du capital social, ou du moins du capital social tel qu'appréhendé par Coleman ${ }^{12}$. Il ne nous semble pas qu'une définition légèrement ultérieure du capital social proposée par Putnam (1993) permette de lever une certaine imprécision de cette notion. Selon ce dernier auteur, le capital social rassemble dans une même notion "les caractéristiques des organisations sociales, telles que les réseaux, les normes, la confiance, qui facilitent la coordination et la coopération pour un bénéfice mutuel".

Il revient à Lin (1995) d'avoir suggéré d'intégrer le capital social, dans une perspective proche de celle que nous défendons, dans une théorie générale des ressources. Lin définit ainsi le capital social comme une richesse potentielle incorporée dans la structure sociale et qui peut être (mais ne l'est pas nécessairement) mobilisée en cas de besoin. Le concept de capital social recouvrirait ainsi à la fois des ressources existantes et des ressources latentes liées à un réseau relationnel stable plus ou moins activé.

11 Voir aussi leur article dans le présent numéro.

12 Cf. notamment N. Sirven (2004).

64 Economie et Institutions $-n^{\circ} 6$ et $7-1^{\text {er }}$ et $2^{\text {e }}$ semestres 2005 
L'activation d'un capital latent nous rapproche ainsi de Hirschman (1986) mais aussi de Bourdieu (1986) pour qui le "capital social se définit par l'ensemble des ressources actuelles ou potentielles qui sont liées à la possession d'un réseau durable de relations plus ou moins institutionnalisées d'interconnaissance et d'interreconnaissance ; ou en d'autres termes à l'appartenance à un groupe comme un ensemble d'agents qui ne sont pas seulement dotés de propriétés communes (...) mais sont aussi unis par des liaisons permanentes et utiles".

Plusieurs auteurs suggèrent cependant la nécessité d'une prise de distance d'avec la notion ou catégorie de capital et, partant, pouvons-nous considérer, celle de capital social. Barthélémy, Nieddu et Vivien (2004) avancent qu'il existe une autre façon de saisir des relations fondamentalement non marchandes qu'en recourant à des concepts le plus souvent non "historicisés" tels que celui "d'externalité" et plus encore ceux exprimés par les différentes extensions de la catégorie "capital" (capital humain, capital social).

S'agissant de l'environnement ou des ressources environnementales, il semble aux auteurs que le "patrimoine", dès lors qu'on le reconnaitrait comme tel, c'est-à-dire que l'on admettrait qu'il est irréductible à du capital, autoriserait à penser les relations économiques de telle façon que le groupe social concerné organise sa perpétuation dans l'espace et dans le temps.

Opposant relations patrimoniales, portant sur des biens à caractère intrinsèquement collectifs et extérieurs au marché, appropriés par un collectif doté d'une dimension intergénérationnelle, et ordre marchand, les auteurs proposent une lecture des politiques agricoles ou encore des politiques environnementales comme des politiques d'arbitrage entre patrimoine et marché.

Nous suivons ces auteurs tout en soulignant que la frontière entre marchand et non marchand, comme celle entre bien public et bien privé ne sont pas données a priori (selon des caractéristiques propres ou immanentes du bien) mais ressortent d'un choix politique (Kaul, 2001).

$\mathrm{Au}$ final, nous considérons que ces deux approches ou entrées, par le capital social et par le patrimoine, ont chacune leur utilité pour saisir les processus d'accumulation et de densification qui sont propres au territoire.

L'approche en termes de capital social, dès lors qu'elle s'intègre dans une théorie générale des ressources, rejoint notre perspective en ce qu'elle souligne l'importance de ressources à caractère latent ou potentiel et met l'accent sur la question centrale de la coordination. Les critiques que l'on peut faire à cette ou à ces approches en termes de capital social sont de deux types. Une première critique, endogène, est qu'elles restent excessivement 
fonctionnelles et souvent mélangent dans un seul concept (le capital) certains attributs des institutions (normes, sanctions), des caractéristiques propres aux individus et à leurs relations (obligation mutuelle, confiance) et certains avantages qui en résultent (circulation de l'information).

La critique exogène à ces approches est celle qui est faite par les auteurs qui proposent de raisonner en utilisant le concept de patrimoine. Le concept de capital serait inapproprié pour appréhender des relations non marchandes.

Nous adhérerions plus aisément à cette critique si au delà de la reconnaissance de la pluralité des ordres, on admettait, comme le suggère Barrère (2004), des ordres incomplets et imparfaits, et par conséquent pluridimensionnels. Il est clair ici que l'insistance avec laquelle nous soulignons l'importance de la proximité institutionnelle ne saurait rimer avec une détermination de celle-ci par le seul ordre marchand et par le seul objectif de réalisation de la promesse de création de richesses contenu dans la proximité organisationnelle.

\subsection{De la nature et de l'utilité de la proximité "géographique"}

Nous avons qualifié le territoire comme "révélé" et comme producteur de ressources, ces qualificatifs s'appuyant sur des coordinations spécifiques. Cela nous conduit à nous interroger sur le caractère ou la dimension proprement géographique de ces coordinations. En d'autres termes, il convient de questionner la nécessité ou même l'utilité de la co-présence physique comme facteur de réalisation de ce que nous avons désigné comme une "promesse" de coordination dès lors que ces ressources sont susceptibles d'être mobilisées.

Nous considérons essentiellement que la proximité "géographique" joue "un rôle" de "facilitateur" de la coordination (B. Pecqueur et J.-B. Zimmermann, 2004).

En ce sens, nous nous démarquons de positions comme celle de $R$. Boschma (2004) ${ }^{13}$ pour qui la proximité géographique ne stimule pas a priori l'apprentissage interactif et l'innovation en ce que cette stimulation serait le fait de réseaux que ceux-ci soient territorialisés ou non.

Cependant, il ne nous semble pas possible non plus, à la différence d'autres chercheurs, de considérer que la proximité "géographique" serait une condition (de la création de ces ressources et de l'accès à un patrimoine) alors que la proximité institutionnelle serait un résultat (le résultat de cet accès de deux personnes au même patrimoine territorial).

13 Voir aussi sa contribution au présent numéro.

66 Economie et Institutions $-n^{\circ} 6$ et $7-1^{\text {er }}$ et $2^{\text {e }}$ semestres 2005 
Enfin, il nous semble difficile de considérer que la proximité "géographique" pourrait être qualifiée de "naturelle"en ce que la proximité "géographique" n'est en rien un rapport direct de l'homme à la nature. Ce rapport, en cela plus justement qualifié de "technique", est toujours un rapport médiatisé par un rapport des hommes entre eux. C'est sans doute pour cette raison que dans les pays dits "développés", les distances sont ainsi désormais de plus en plus données en temps et non en mesure métrique physique.

La proximité "géographique" tient aujourd'hui principalement, selon nous, du rapport des hommes entre eux.

Elle ne peut donc pas être considérée principalement et a fortiori exclusivement comme une condition de ce rapport, et l'on peut au moins tout aussi valablement la considérer comme un résultat de la coordination des acteurs.

De manière corollaire, la proximité institutionnelle, si elle peut être appréhendée comme un résultat, peut également être considérée comme une condition. C'est parce qu'elles parlent la même langue (celle pratiquée ou associée à un territoire), partagent certaines représentations, que deux personnes vont coopérer et ainsi révéler ou créer certaines ressources. La notion de "structure sociale" (Giddens, 1987) prend ici tout son sens en ce qu'elle combine ou associe les règles par lesquelles s'opère toute coordination et les ressources qui sont mobilisées. Il n'est pas nécessaire selon nous de poser que la structure serait le "conteneur" de ces dernières pour ne pas confondre règles et ressources. La définition de Giddens de la structure ou, plus précisément, de "l'ensemble structurel" comme "formé par la convertibilité réciproque des règles et des ressources engagées dans la production sociale" est ici suffisante et pleinement satisfaisante.

Nous considérons donc que la proximité "géographique" et la proximité institutionnelle sont à la fois un résultat et une condition, ce qui nous semble conforme à la fois à une approche en dynamique de la question de la coordination "située" et à une compréhension de la proximité comme condition et effet.

Cette dernière définition met d'abord l'accent sur la proximité "géographique", tout en reconnaissant ou en indiquant que celle-ci est "structurée" par des institutions. Plutôt que "structurée" par des institutions, la proximité géographique est, en fait, selon nous, ellemême un résultat institutionnel. Au point que l'on pourrait se demander si cette catégorie (géographique) a encore lieu d'exister autrement que sur un plan analytique...

La réponse est positive car, comme nous l'avons dit, il n'y aurait aucun sens à parler ici de territoire sans proximité géographique. Cependant, c'est bien selon nous le temps qui produit l'espace, et non l'inverse (Colletis et Rychen, 2004). 
Le "besoin" de proximité géographique, le "face-à-face" en d'autres termes (question qui ne se confond pas avec celle, dont la réponse est évidente, de la nécessité de la proximité géographique pour que l'on puisse parler de processus de construction territoriale), réside sans doute en grande partie dans ce qui fait la différence entre circulation de l'information et production de connaissance. Comme ceci a été maintes fois souligné, autant la première n'impose pas, tant s'en faut, la proximité "géographique", autant la seconde (production de connaissance) semble utile, voire indispensable ou du moins nécessaire pour révéler et combiner des savoirs tacites, par essais, erreurs et tâtonnements, lorsqu'il s'agit de résoudre un problème alors que ses fins (ou issues) et les moyens à mobiliser (ressources cognitives) ne sont pas connus à l'avance.

\subsection{Les trois dimensions de la proximité en interaction}

Nous souscrivons à la thèse de Kirat et Lung (1995) lorsque ceux-ci écrivent que "les organisations constituent un espace de définition des pratiques et des stratégies des agents à l'intérieur d'un ensemble de règles porté par les institutions". Nous estimons "qu'il n'y a pas de proximité organisationnelle génératrice de coordination sans une certaine dose de proximité institutionnelle, indispensable à la coordination d'agents interdépendants mais dotés d'un degré plus ou moins élevé d'autonomie décisionnelle" (Pecqueur et Zimmermann, 2004).

De la même manière que les relations entre ressources et actifs d'une part, facteurs génériques et spécifiques d'autre part, sont réversibles (activation et désactivation, spécification et déspécification étant toujours possibles), les relations entre les différentes dimensions de la proximité sont variables et réversibles.

Ceci signifie que dans tel processus de révélation du territoire, c'est plutôt la proximité spatiale qui domine les deux autres (organisationnelle et institutionnelle) alors que dans tel autre, la combinaison d'intensité des trois dimensions de la proximité est différente, la proximité organisationnelle ou institutionnelle pouvant à leur tour dominer.

L'hypothèse avancée dans un autre texte (Colletis et Rychen, 2004) est que le développement local en termes "d'agglomération" fait principalement appel à la proximité géographique, que celui en termes de "spécialisation" suggère d'abord une proximité organisationnelle forte, enfin, que le développement en termes de "spécification" repose avant tout sur la proximité institutionnelle. Mais on comprendra que ces distinctions n'ont de sens qu'en dynamique et en porosité de ces différents modes. Ainsi, à titre d'illustration, une dynamique d'agglomération (dominée par la proximité spatiale ou géographique) contient les éléments 
susceptibles de déboucher sur un développement en termes de spécialisation ou de spécification. Dans ce cas, des ressources devront être activées dans un certain sens : celui d'une complémentarité sectorielle ou technologique, par exemple, impliquant à la fois une proximité organisationnelle et une proximité institutionnelle particulières.

\section{Conclusion : une nouvelle perception de la concurrence entre les territoires}

La notion de "concurrence entre les territoires" est une notion qui peut suggérer une concurrence entre territoires postulés ou existants comme tels, c'est-à-dire associant une dotation particulière à un territoire circonscrit, bien identifié dans l'espace.

Comme on l'aura compris, les "facteurs de concurrence spatiale" que nous proposons de retenir relèvent d'une autre représentation des processus de construction territoriale.

Cette représentation met l'accent sur la question décisive de la coordination ainsi que sur celle de la création de ressources.

La capacité de production de ressources spécifiques peut constituer un avantage productif dans la concurrence de territoires à géométrie variable, se définissant non pas en rapport à des frontières, mais en lien avec un patrimoine tel que nous l'avons appréhendé, c'est-à-dire articulant de manière non mécanique et en fonction d'une architecture en permanente redéfinition le passé et l'avenir possible.

Ce patrimoine n'est pas un capital. Il est encore moins un stock de ressources disponibles en l'état. Il n'a, en effet, de valeur que contextuelle.

Comme nous l'avons écrit, les seules ressources dont un territoire dispose (et non contient) sont des ressources liées à une trace d'activités de coordination passées (mémoire, confiance) et à un potentiel ou à une virtualité de nature cognitive (ressources cognitives virtuellement complémentaires).

Ici, et sans nullement nous inscrire dans une appréhension de territoires comme si ceux-ci existaient comme tels, il est possible d'observer une grande diversité de configurations spatiales en termes de processus de construction territoriale.

C'est précisément cette diversité qui est suggérée lorsque, avec d'autres ${ }^{14}$, nous proposons de distinguer trois modes de développement local : l'agglomération, la spécialisation, et la spécification. Avec les mêmes, nous avons indiqué que chacun de ces trois modes fait appel à une combinaison particulière des trois

14 G. Colletis, J.-P. Gilly et al. (1999). Cet article se fonde très largement sur une étude coordonnée par J.-B. Zimmermann pour le Commissariat Général du Plan (Zimmermann, coord., 1998). 
dimensions de la proximité (géographique, organisationnelle, institutionnelle).

Plutôt que de raisonner la concurrence entre les territoires en termes de "protection face aux aléas de marchés mondiaux", il convient de penser la construction de trajectoires territoriales en fondant celles-ci sur la production de ressources cognitives spécifiques comme facteur d'enrichissement et de viabilité d'un patrimoine territorial sans cesse redéfini en fonction du passé et des nouveaux problèmes à résoudre.

Le passage allant de la spécialisation entre des nations en fonction de leur dotation factorielle à la différenciation entre les territoires passe par une réflexion en des termes nouveaux de ce sur quoi se fonde "l'avantage comparatif".

L'avantage comparatif ricardien met en jeu des facteurs (de facto) génériques qui sont comparés sur la base des coûts et des prix d'un pays à l'autre. Ceci induit un schéma de spécialisation fondé tantôt sur une mobilité des produits (échanges de produits ou de services dans le commerce international), tantôt sur une mobilité de facteurs de production "classiques" (l'investissement direct et, dans une moindre mesure, les migrations internationales). Dans les deux cas, la spécialisation est expliquée par le jeu du prix des facteurs et ne tient que peu ou pas compte de dynamiques qui peuvent être de nature à attribuer aux ressources ou à la production de celles-ci une valeur hautement contextuelle, difficilement redéployable ou transférable, et ne se prêtant pas aux comparaisons ordinales.

Nous rejoignons ici la notion de ressources spécifiques que nous avons évoquée dans ce texte et des textes précédents (op. cit.). En effet, la spécificité "cloue" la ressource à son contexte productif et/ou territorial.

L'avantage comparatif ne se joue plus dès lors sur une gamme ordinale de productivité comparée, le caractère spécifique de l'actif ou de la ressource rompant avec ce type de comparabilité. On ne peut donc parler, à propos des actifs et ressources spécifiques, d'avantages comparatifs mais plutôt d'avantages "différenciatifs" qui supposent pour être produits ou valorisés des stratégies radicalement différentes de celles qui visent à susciter ou conforter des avantages comparatifs.

Le modèle de concurrence proposé est différent du modèle ricardien de dotation-spécialisation en ce que les perspectives de création de richesses tiennent ici aux capacités de groupes à s'organiser et à élaborer des processus originaux d'émergence des ressources.

La mondialisation, qui consiste essentiellement en l'interconnexion des marchés (surtout financiers) et qui crée des liens de cause à effet de plus en plus denses entre les acteurs 
économiques, produit en même temps des dynamiques et des procédures singulières de création de ressources.

C'est pourquoi il nous semble que l'analyse des processus concomitants de production, révélation ou valorisation de ressources nouvelles, et de spécification des territoires, c'est-à-dire l'analyse des rapports particuliers qu'entretiennent entre eux les hommes qui vivent dans ces territoires, constitue probablement un chantier crucial de l'analyse économique des années qui viennent.

Cette analyse renvoie aux trois questions principales des ressources d'autorité, de la nature des principales externalités produites (sans doute ni pécuniaires ni même technologiques mais de type "culturel"), des dispositifs, enfin, et modes de coordination (reposant d'abord sur les institutions).

\section{Références}

Abdelmalki L., D. Dufourt, Th. Kirat et D. Requier-Desjardins (1996), "Technologie, institutions et territoires : le territoire comme création collective et ressource institutionnelle" in B. Pecqueur (dir.), Dynamiques territoriales et mutations économiques, Paris, L'Harmattan.

Audretsch D.-B. et M.-P. Feldman (1996), "Innovative Clusters and the Industry Life Cycle", Review of Industrial Organization, 11, pp. 253-273.

Aydalot P. (1985), Economie régionale et urbaine, Paris, Economica. Barrère C. (2004), "Le dualisme des ordres de la modernité" in Patrimoine. Ordres et dynamique du capitalisme, Géographie, Economie, Société, $\mathrm{n}^{\circ} 3$.

Barrère C., D. Barthélémy, H. Ollagnon, M. Nieddu, F.-D. Vivien (2001), "Patrimoine, approches croisées", Ecole doctorale des Sciences juridiques, économiques et de gestion, Université de Reims, Cahier $\mathrm{n}^{\circ} 2$, mars 2001.

Barrère C., D. Barthélémy, M. Nieddu, F.-D. Vivien (dir.) (2005), Réinventer le patrimoine. De la culture à l'économie, une nouvelle pensée du patrimoine ? Paris, L'Harmattan.

Barthélémy D., M. Nieddu et F.-D. Vivien (2004), "Externalités ou production de patrimoine ? Les enseignements de travaux récents sur l'agriculture et l'environnement", dans Patrimoine. Ordres et dynamique du capitalisme, Géographie, Economie, Société, n³.

Becattini G. (1979), "Dal Settore industriale al Distretto industriale. Alcune Consideratione sull'Unita d'Indagine dell'Economia industriale", Revista di Economia e Politica industriale, $\mathrm{n}^{\circ} 1$.

Benko G. et A. Lipietz (2000), La richesse des régions, Paris, PUF.

Billaudot B. (2004), "Proximité, réseaux et coordination industrielle : quelle articulation conceptuelle ?" Quatrièmes Journées de la Proximité, Marseille, 17-18 juin 2004. 
Boschma R. (2004), "Proximité et innovation", Economie Rurale, $\mathrm{n}^{\circ} 280$, mars-avril, pp.8-24.

Bourdeau-Lepage L. et J.-M. Huriot (2003), "The Metropolis in Retrospect. Permanence and Change", LIIe Congrès de l'AFSE, Paris, 18-19 septembre 2003.

Bourdieu P. (1986), "The forms of capital", in J.G. Richardson (Ed.), Handbook of Theory and Research for the Sociology of Education, New York, pp.241-258.

Camagni R. (1999), avant-propos de l'ouvrage collectif : Les milieux urbains : innovation, systèmes de production et ancrage, IRERGREMI, Neuchâtel, pp.1-5.

Coleman J. (1988), "Social Capital in the Creation of Human Capital", American Journal of Sociology, vol. 94, pp. 95-120.

Colletis G. (1998), "Intelligence économique et développement territorial", Revue d'Intelligence économique, $\mathrm{n}^{\circ} 3$, oct.

Colletis G. et B. Pecqueur (1993), "Intégration des espaces et quasiintégration des firmes : vers de nouvelles rencontres productives ?" dans M. Bellet, G. Colletis et Y. Lung, Economies de proximités, Revue d'Economie Régionale et Urbaine, ${ }^{\circ} 3$, sept.-oct., pp. 489-508.

Colletis G. et B. Pecqueur (1995), "Les facteurs de concurrence spatiale", communication au colloque Dynamiques industrielles et dynamiques spatiales, Toulouse, 30, 31 août et $1^{\text {er }}$ septembre 1995.

Colletis G. et F. Rychen (2004), "Entreprises et territoires : proximités et développement local", Pecqueur B. et J.-B. Zimmermann (dir.) (2004), Economie de proximités, Paris, éditions Hermès-Lavoisier.

Colletis G., B. Pecqueur et J.-P. Gilly (2001), "Inscription spatiale des firmes, gouvernance des territoires et régulation d'ensemble", Séminaire Régulations sectorielles et territoriales.

Colletis G., J.-P. Gilly et al. (1999), " Construction territoriale et dynamiques économiques", Sciences de la Société, n48, octobre, pp.25-47.

Cooke P. et K. Morgan (1998), The Associational Economy: Firms, Regions and Innovation, New York, Oxford University Press.

Courlet C. (2001), Territoires et régions : les grands oubliés du développement économique, Paris, L'Harmattan.

Gay C. et F. Picard (2001), "Innovation, agglomération et espace : une mise en perspective de la littérature", Economies et Sociétés, série W Dynamique technologique et organisation, $n^{\circ} 6,4 / 2001$, pp. 679-716. Giddens A. (1987), La constitution de la société, Paris, PUF.

Guillain R. et J.-M. Huriot (2000), "Le rôle agglomératif des externalités d'informations, mythe ou réalité ?" dans C. Baumont et al. (dir.), Economie géographique : les théories à l'épreuve des faits, Paris, Economica.

Hirschman A.-O. (1986), Vers une économie politique élargie, Paris, Editions de minuit. 
Kaul I. (2001), "Public Goods: Taking the Concept to the $21 \mathrm{st}$ Century", in D. Drache (Ed.), The Market of the Public Domain, London-New York, Routledge, pp.255-273.

Kébir L. (2004), Ressource et Développement : une approche institutionnelle et territoriale, Thèse de Sciences économiques, Université de Neuchâtel.

Kirat T. et Y. Lung (1995), "Innovation et proximités : le territoire, lieu de déploiement des processus d'apprentissage", dans N. Lazaric et J.-M. Monnier (dir.), Coordination économique et apprentissage des firmes, Paris, Economica.

Lancaster K. (1991), Modern Consumer Theory A new approach of Consumer Theory, Edward Elgar Publ. Company.

Lévy J. et M. Lussault (2003), Dictionnaire de la géographie et de l'espace des sociétés, Paris, Belin.

Lin N. (1995), "Les ressources sociales : une théorie du capital social", Revue française de sociologie, ${ }^{\circ} 36$, pp. 685-704.

Massard N., A. Torre et O. Crevoisier (2004), "Proximité géographique et innovation", dans Pecqueur B. et J.-B. Zimmermann (dir.), Economie de proximités, Paris, éditions Hermès-Lavoisier.

Mathis, J., J. Mazier et D. Rivaud-Danset (1988), La compétitivité industrielle, Paris, Economica.

Olivier V. et F. Wallet (2004), "Filières agro-alimentaires localisées et développement territorial : à quoi sert la notion de proximité dans l'analyse des rapports entre acteurs ?" Quatrièmes Journées de la Proximité, Marseille, 17-18 juin 2004.

Pecqueur B. (2002), "Dans quelles conditions les objets patrimoniaux peuvent-ils être un apport d'activités ?", Revue Montagnes méditerranéennes, $\mathrm{n}^{\circ} 15$.

Pecqueur B. et J.-B. Zimmermann (dir.) (2004), Economie de proximités, Paris, éditions Hermès-Lavoisier.

Pecqueur B. et V. Peyrache-Gadeau (2002), "Les ressources patrimoniales : une modalité de valorisation pour les milieux innovateurs de ressources latentes ou existantes", Colloque GREMI, 26-27 mai 2002, Neuchâtel.

Piore M. et Ch. Sabel (1984), The Second Industrial Divide, New York, Basic Books [trad.fr. Les chemins de la prospérité, 1988].

Putnam R.D. (1993), Making Democracy Work: Civic Traditions in Modern Italy, Princeton University Press.

Rémy J. (1966), La ville, phénomène économique, Editions sociales [2ème édition 1999, Economica, Paris].

Sassen S. (1991), The Global City, Princeton University Press.

Scott A.-J. (2001), Les régions et l'économie mondiale, Paris, L'Harmattan.

Sirven N. (2004), Capital social et développement : concept, théories et éléments empiriques issus du milieu rural de Madagascar, Thèse pour le doctorat d'économie, Université de Bordeaux 4. 
Veblen T. (1899), La théorie de la classe de loisir, Paris, Gallimard [trad.fr. 1970].

Zimmermann J.-B. (coord.) (1998), Construction territoriale et dynamiques productives, Convention d'étude $n^{\circ} 18 / 1997$ du Commissariat Général du Plan, Rapport final, décembre.

74 Economie et Institutions $-n^{\circ} 6$ et $7-1^{\text {er }}$ et $2^{\text {e }}$ semestres 2005 